\title{
LA FORMACIÓN DOCENTE PARA LAS INFANCIAS. UN APORTE DESDE LAS NARRACIONES DE FORMACIÓN DE GRADUADAS EN LA UNIVERSIDAD
}

\section{TEACHER TRAINING FOR CHILDHOODS. A CONTRIBUTION FROM THE NARRATIVES OF GRADUATE TRAINING AT THE UNIVERSITY}

Ileana Ramírez $^{1}-$ Vanina Soledad Borda $^{2}$

Fecha de recepción: 12-06-2020

Fecha de aceptación y versión final: 20-11- 2020

\section{Resumen}

Este artículo presenta avances de una investigación cuyo propósito es el estudio de los sentidos sobre la formación docente que distintos protagonistas construyen. Para esta ocasión presentaremos unas lecturas sobre del trabajo que realizamos con graduadas de la carrera de Profesorado en Educación Inicial, de una Universidad pública. Nuestra investigación asume una perspectiva de análisis cualitativa, en tanto se enfoca en la mirada de las protagonistas, buscando establecer acontecimientos significativos que surgen de sus narraciones de formación. Desde allí, se propone una descripción y reflexión sobre la formación docente. De los relatos de las graduadas, identificamos algunos ejes de análisis: los motivos para el ingreso a la formación docente y las expectativas sobre la carrera, los acontecimientos significados como obstaculizadores en el trayecto de formación y, por último, acontecimientos que conmovieron de manera positiva la experiencia de formación y la comprensión del futuro rol profesional. Finalmente, cabe mencionar que, este trabajo se enmarca en una investigación en curso, que tiene como objetivo estudiar la formación docente para las infancias, proponiendo dos tipos de lecturas. Por un lado, una mirada histórica que nos acerca a los sentidos fundacionales del Profesorado en Educación Inicial en la Universidad, y otra lectura en clave pedagógica, que recupera las experiencias de diferentes actores en el marco de dicha carrera.

Palabras clave: formación docente- graduadas- narraciones de formación- universidad.

\section{Abstract}

This article presents advances of an investigation whose purpose is the study of the senses on the teacher training that different protagonists construct. For this occasion, we will present some readings on the work we do with graduates of the Teaching career in Initial Education, from a public University. Our research assumes a qualitative analysis perspective, as it focuses on the gaze of the protagonists, seeking to establish significant events that emerge from their training narratives. From there, a description and reflection on teacher training is proposed. From the stories of the graduates, we identify some axes of analysis: the reasons for entering teacher training and expectations about the career, the events that are meant as obstacles in the

\footnotetext{
${ }^{1}$ Profesora en Ciencias de la Educación. Diplomada en Pedagogía de las Diferencias, FLACSO. Esp. en Docencia Universitaria, UNNE. Doctora en Educación, UNER. Docente del departamento en Educación Inicial de la Facultad de Humanidades, UNNE. Resistencia, Chaco - República Argentina. Correo electrónico: ileana.ramirez@ comunidad.unne.edu.ar

${ }^{2}$ Profesora en Educación Inicial. Estudiante de la Diplomatura Pedagogía Hospitalaria y Domiciliaria. Tesista de la Licenciatura en Educación Inicial de la Facultad de Humanidades, UNNE. Resistencia, Chaco - República Argentina. Correo electrónico: bordavanina.18@gmail.com
} 
training journey, and finally, events that positively affected training experience and understanding of the future professional role. Finally, it is worth mentioning that this work is part of an ongoing investigation, which aims to study teacher training for childhood, proposing two types of readings. On the one hand, a historical look that brings us closer to the foundational meanings of the Teaching Staff in Initial Education at the University, and another reading in a pedagogical key, which recovers the experiences of different actors within the framework of said career.

Key words: teacher training- graduates- training narratives - university.

\section{Introducción}

En este artículo presentamos avances de un trabajo realizado con graduadas de la carrera de Profesorado en Educación Inicial, de la Facultad de Humanidades de la Universidad Nacional del Nordeste (UNNE). Nuestro propósito es poder conocer y comprender las características que asume la formación docente para la infancia en un contexto universitario, y los sentidos que construyen las graduadas a partir de su experiencia de formación.

Adoptamos una perspectiva cualitativa de la investigación. Desde allí proponemos una lectura de las experiencias de formación de las entrevistadas, narradas a partir del trabajo en entrevistas en profundidad. Las mismas se realizaron después de un tiempo de haber finalizado la carrera de formación docente. Por lo tanto, las entrevistadas no tienen una antigüedad mayor a cinco años de su egreso. Sus experiencias de formación no son tan lejanas, "pero en su lugar de 'contadoras' pueden distanciarse de ella porque ya no son parte de la misma" (Rivas Flores y Leite, 2014 , p. 61). De allí que entendemos que sus relatos contienen sentidos otorgados a determinados acontecimientos que son narrados como significativos en el tiempo de formación transitado.

Cabe mencionar que esta presentación se enmarca en un proyecto de investigación denominado "La formación docente para la educación infantil en la Universidad. Lecturas entre el pasado y el presente". Desde allí se propone el estudio de la formación docente en Educación Inicial desde dos lecturas. La lectura histórica, que busca conocer los sentidos o mandatos fundantes en torno a la carrera de Profesorado en Educación Pre- Elemental, en la década del 70 en la Facultad de Humanidades de la UNNE, recuperando sentidos de formación, referentes e instituciones paradigmáticas, en Resistencia Chaco, pero siempre pensando desde el anclaje al contexto mayor, que es nuestro país.

La lectura pedagógica, que busca situar la mirada en un presente para comprender cuáles son y cómo se van construyendo los sentidos sobre la formación docente que elaboran algunos/as de sus protagonistas.

\section{Respecto de nuestras decisiones metodológicas}

Nuestro estudio asume una perspectiva de análisis cualitativa, ya que, como expresa Vasilachis de Gialdino (2006) pretende la valoración y el intento por descubrir los sentidos sobre la formación docente que graduadas del profesorado de Educación Inicial han construido a partir de sus experiencias de formación en el contexto de la 
Universidad. Por lo tanto, es "un proceso interactivo entre el investigador y los participantes" (p.27).

Por otra parte, teniendo en cuenta que nos enfocamos en la mirada de las protagonistas, buscando establecer las relaciones significativas que surgen a partir de los acontecimientos narrados, se tomará como procedimiento metodológico el enfoque biográfico-narrativo. Éste permitirá "construir sentido, por medio de la descripción y análisis de los datos biográficos" (Bolívar, 2001, p. 20) de las entrevistadas. Dicho abordaje nos posibilita "comprender lo singular/universal de las historias, memorias de formación, al revelar prácticas individuales/colectivas inscriptas en la itinerancia de los sujetos en el aprendizaje y formación" (De Souza, 2008, p. 6).

Desde este contexto, trabajamos con narrativas de formación. Entendemos a las mismas como el relato que las entrevistadas, en este caso las graduadas, realizan de su experiencia de formación. Estas narrativas, constituyen un elemento central en esta investigación, siendo "potencializadoras para la comprensión del proceso de formación” (De Souza, 2008, p. 5).

En 2019, realizamos entrevistas en profundidad a dos graduadas de la carrera de Profesorado en Educación Inicial, de la Facultad de Humanidades de la UNNE. Las mismas no tienen más de cinco años de finalizado el profesorado.

Cabe señalar que se tuvo en cuenta casos extremos, con el supuesto de que los mismos podían ofrecer variedad y, retomando a Sirvent (2005), tensionar al "máximo las diferencias". Por consiguiente, un criterio que consideramos fue el contexto social y cultural de las graduadas, ya que posibilita pensar el acceso al capital cultural y su relación con la etapa de formación inicial. Así también, la edad y duración de la carrera, fueron otros factores a tener en cuenta a la hora de la elección. De allí que nuestra muestra se conformó de la siguiente manera:

La Graduada 1 (G1) decide estudiar Educación Inicial, evaluando entre dos opciones: Psicología (rama Infancia) y Educación Inicial. Uno de los motivos que la llevó a dicha opción fue la cercanía de la Facultad y el gusto por la educación y la infancia, fundado en los doce años de haber cursado en un colegio confesional con cierta premisa orientada a la educación hacia los más necesitados. En paralelo a esta elección comienza a trabajar en un colegio confesional en el área de catequesis, con edades entre cuatro y seis años.

G1 ingresó a la carrera en el año 2014 y finalizó según los tiempos estipulados en el plan de estudios (cuatro años).

La Graduada 2 (G2) empezó la carrera porque quería "ser alguien" (es la única en su familia que estudió una carrera universitaria). Viene de una familia de bajos recursos. Su madre es empleada doméstica y esta condición la marcó, en el sentido de creer que no podía ser profesional.

G2 inició la carrera en 2011 y la duración fue de siete años. Dejó el trabajo de auxiliar de jardín para comenzar a estudiar el profesorado en Educación Inicial. Durante el cursado fue madre y desarrolló distintos tipos de trabajos para solventar sus gastos, como venta de ropa, cosméticos, entre otros.

La perspectiva metodológica que asumimos nos permite, inicialmente, entender los sentimientos y representaciones de los actores sociales en sus procesos de 
formación (De Souza, 2008), también avanzar en la comprensión del lugar que la subjetividad tiene como constitutiva del quehacer pedagógico y, al decir de Sverdlick (2007) "de la producción del conocimiento colectivo" (p. 21). Es, en este sentido, que compartimos con Rivas Flores y Leite (2014) que:

En este juego dialéctico se van configurando las identidades (Rivas, et. al., 2010), como un modo particular de leer y comprender el marco institucional en el que se actúa. De esta forma, el interés por lo biográfico como estrategia de investigación, no es sino el intento de comprender mejor la sociedad en que vivimos, y de la escuela en especial, desde la peculiar perspectiva de cada uno de los sujetos que forman parte de ella. A pesar de que consideramos que cada experiencia en sí misma es relevante, nuestra finalidad no es tanto el propio sujeto sino el modo en que éste ha construido su visión sobre la escuela $y$, de este modo, cómo ha desarrollado su peculiar modo de actuación en la misma. (p. 60).

\section{La formación docente, un camino revisitado}

Partimos de considerar que la formación no queda supeditada a la adquisición de ciertos contenidos teóricos o comportamientos profesionales, elegimos pensarla, también, como un proceso de construcción complejo, personal, que toma rumbos diferentes según cada sujeto. En este sentido, Alliaud (2010) expresa que no está formado quien encara mejor la forma o el formato de otros, sino quien utiliza los modelos como una posibilidad, como un ejemplo que le permita armar o al menos encarar su propia producción, al igual que un artista. Para la autora, la formación puede ser vista como un relato de experiencias "marcadas con la huella del que narra y experimenta" ( $p$. 151), con un saber que tiene unas características particulares, porque es subjetivo, vinculado a la maduración de un individuo.

En sintonía con lo planteado, recuperamos los aportes de Masschelein y Simons (2014) quienes nos permiten pensar a la formación como orientación hacia el mundo, como una apertura a conocer lo 'nuevo' que se nos presenta mediante unos contenidos, unas materias, unas estrategias. Dirán estos autores que la formación conlleva transformación, "Así pues la formación implica salir constantemente de uno mismo o trascenderse a sí mismo: ir más allá de sí mismo y del propio mundo vital por medio de la práctica y del estudio" (p. 22).

Pensamos también que, en la conjunción de una propuesta de formación, materializada en un plan de estudios, y la experiencia de formación vivida por cada protagonista, se pueden identificar algunos acontecimientos que dan cuenta de la transformación que cada uno, que cada una realiza.

Asimismo, avanzamos junto con Larrosa (2000) en comprender que, en el trayecto formativo se configuran una serie de etapas. La primera, se corresponde con el ingreso a la Facultad, y específicamente a la formación docente. Ésta constituye una fase de afianzamiento, entiéndase como ruptura o refuerzo de las concepciones con las cuales se ha ingresado a la carrera. La segunda es un momento de desequilibrio donde lo antes construido corre el peligro de destruirse, es un momento que obliga a tomar decisiones sobre cómo seguir transitando. Y, por último, una etapa de resignificación del recorrido formativo, en el cual las graduadas se posicionan en un lugar de afirmación de la identidad docente. 
Desde este punto, la formación puede entenderse como una experiencia movilizadora y configuradora de una nueva forma, un camino que se reinventa cada día, en el cual el tiempo de formación no es un tiempo lineal ni pendular, es decir de ida y vuelta, sino más bien, un "movimiento que conduce a la confluencia en un punto mágico de una sucesión de círculos excéntricos" (Larrosa, 2000, p. 79).

Este aporte de Larrosa, permite pensar en cada etapa como una sucesión de círculos que se originan en un punto en común. Para que cada círculo dé lugar a otro, es necesario un descentramiento que genera la apertura a una nueva etapa. Esta apertura sólo puede darse cuando el círculo vuelve al momento de origen. Por lo tanto, la primera etapa podría considerarse como el primer círculo, el que da origen a los otros dos. Es el círculo de la forma, la seguridad, del primer contacto con este camino de formación del cual se generan las primeras experiencias en relación con la carrera. El segundo círculo, corresponde a la segunda etapa mencionada del recorrido formativo. Podríamos tomar este nuevo espacio como la deformación, la incertidumbre, un momento de desequilibrio que conduce a las promesas de la transfiguración (Larrosa, 2000).

Por último, el tercer círculo nos coloca ante casi el final de la carrera, la esperanza de una nueva manera de relacionarse con la futura profesión. Es la transfiguración, el encuentro con ese deseo primero, es el momento de volver al inicio de las aperturas, al primer círculo y re-significarlo. Empezar a vivir la propia identidad docente desde lo que se es y se fue construyendo en este proceso de formación.

El sujeto, en el proceso de formación, toma decisiones sobre un trayecto formativo organizado como lo es una carrera de formación docente, y al que le van sucediendo acontecimientos que impactan de distintas maneras.

Al acontecimiento lo pensamos como un gesto o acción, que al ser consciente y estar cargado de sentido, nos transforma (Toro, 2005). Nuevamente recurrimos a Masschelein y Simons (2014) que plantean que el acontecimiento es,

como algo que nos hace pensar, que despierta nuestro interés, que hace que algo se torne real y significativo, que se convierta en una materia o en un asunto que importa. Una demostración matemática, una novela, un virus, un cromosoma, un bloque de madera o un motor: todas estas cosas se vuelven interesantes y significativas. (p.23)

A lo planteado, nos interesa agregar que, un acontecimiento, entonces, marca un antes y un después y nos instala en un presente (Bárcena y Mélich, 2014). El acontecimiento es, además, una determinada experiencia de la vivencia del tiempo. Esto significa, entonces, que el mismo solo se explica como una ocasión, como estado de decepción, como lo imprevisible, como un instante del todo original, como aquello que irrumpe por sorpresa y que resquebraja la continuidad del tiempo (Borda y Ramírez, 2019).

\section{3.a. Rasgos de identidad de la formación docente para la educación infantil}

En Argentina, hay que reconocer la centralidad que la educación primaria ha tenido en la configuración y desarrollo de la formación docente. A través de su institución paradigmática, la Escuela Normal Nacional, marcó una época fundacional en el sistema educativo. Pero el recorrido de formación vinculado al trabajo con los 
jardines de infantes estuvo signado por otros sentidos, referimos al histórico debate sobre el carácter educativo o doméstico del jardín de infantes, (Carli, 2002; Diker y Terigi, 2003; Ponce, 2012), que repercutió en las decisiones políticas en torno a la formación de docentes para el nivel inicial,

esta polémica sobre la función del nivel réplica en debates acerca del peso que corresponde, en los planes de estudio, a la formación llamada 'pedagógica' (centrada en la función educativa del jardín) frente a una capacitación de tipo puramente asistencial (centrada en el entretenimiento y el cuidado de los niños). (Diker y Terigi, 2003, p. 46)

En el marco de estas discusiones es que queremos seguir pensando sobre la formación docente para el trabajo con las infancias, y específicamente, cómo se fue definiendo en el contexto universitario.

En trabajos anteriores (Musin, Ramirez y Almirón, 2016), hemos dado cuenta de los sentidos fundantes de la formación docente para las infancias en el contexto de la Facultad de Humanidades de la UNNE. A partir del análisis del primer plan de Estudios de la carrera Profesorado en Educación Pre- Elemental, en el año 1973, identificamos una impronta de la didáctica y de la psicología, con la presencia casi exclusiva de la psicología de corte experimental- natural. Las prácticas y pasantías acompañaban todo el proceso de formación. El lugar de las prácticas, se ha ido resignificando en los sucesivos planes de estudios, pero nunca ha perdido centralidad.

En el plan de estudios vigente, aprobado por Res. № 739/99-CS- UNNE, identificamos como un rasgo de identidad, la presencia de los Talleres de Investigación, Integración y Práctica, un taller por año de duración de la carrera. Cuentan con instancias presenciales y de trabajo de campo, intentando de esta manera, borrar los límites que existen entre "teoría" y "práctica" en la formación (organización propia de la estructura academicista). El trabajo de campo implica, a su vez, el ingreso de las y los estudiantes a instituciones educativas. Esto es una característica que nos permite decir que, en este trayecto de formación, desde el primer año de la carrera, los y las estudiantes ya tienen contacto con el futuro campo laboral, siendo una continuidad con el periodo fundacional de la carrera.

\section{Las huellas en el camino. Acontecimientos significativos en la formación}

En este punto, presentaremos algunos planteos analíticos en torno a unos ejes que construimos a partir de identificar acontecimientos significativos, planteados en las narraciones de formación de las graduadas. En dichas narraciones se expresa la peculiar manera en que cada una de ellas ha construido su visión sobre la formación docente y sobre la formación en el contexto de una Universidad pública.

Los temas que aparecen como centrales, y que organizan este apartado, se vinculan con las expectativas y los motivos al momento de ingresar a la formación docente para la educación infantil; los acontecimientos vividos como obstáculos en el camino, como así también, aquellos que fueron significados como positivos y que han dejado una huella. 


\section{4.a. Sobre motivos y expectativas en el ingreso a la formación docente para la Educación Inicial}

Sin dudas que el ingreso a una carrera de formación docente, como a cualquier otra, es una decisión que sostiene un proyecto de vida. Cada proyecto personal, toma impulso por motivos subjetivos, propios, pero que dan cuenta de una historia familiar, socioeducativa, laboral. Sandra Carli (2012) nos ayuda a pensar las expectativas y las decisiones en torno a la elección de la carrera, teniendo en cuenta que las influencias, "no (son) como un argumento explicativo que indicaría el peso unidireccional de un elemento sobre otro, sino como aquello que expresa la trama de intersubjetividad en la que toman forma las decisiones "individuales" (p.85).

En una de las entrevistadas, el motivo central por el cual decide iniciar la formación docente es el desafío personal, de superación de una autoprofecía construida en su historia familiar, desde la cual sus posibilidades se percibían como limitadas. De allí que el ingreso a la Universidad representó romper sus propios límites, como mujer de un sector social determinado y como estudiante que atravesó otras experiencias de formación que resultaron frustrantes. G2 relata:

vengo de una historia de bajos recursos (...) siempre me trataron de que yo no puedo hacer nada porque yo soy la hija de la empleada doméstica, entonces yo como voy a poder ir a la escuela, cómo voy a poder ser profesional.

Entre los motivos asoman con fuerza, en ambos casos, el hacer una carrera en el ámbito de una universidad. Además, se menciona la decisión de trabajar con la población infantil.

En este punto, identificamos sentidos relacionados con una representación sobre el profesorado y sobre el ser docente de Educación Inicial. Aparece la figura de la "maestra jardinera", desde una mirada idealizada que enuncia como condición, casi ineludible para el ejercicio de esta profesión el "soy muy maternal con los niños" (G1). Construyéndose de este modo, la imagen de una mujer alegre, cariñosa y que, sin dudas, profesa amor por los niños. Se argumentan estos sentidos al actualizar el recuerdo de sus maestras jardineras, enfatizando en la calidez que rememoran en dichas docentes.

Observamos aquí cómo ciertas experiencias, algunas comunes, contribuyen a conformar una especie de sentido común pedagógico, que se manifiesta en una serie de actitudes y valoraciones compartidas acerca de lo que significa ser docente de Educación Inicial, y también en el tipo de formación específica que esperan encontrar.

Respecto de las expectativas sobre la carrera, las entrevistadas coinciden en que, al momento de tomar la decisión de ingresar al profesorado de Educación Inicial, no conocían de qué se trataba. Una de ellas nos comenta:

vos empezás la Facultad y no tenés vida (...) es muy demandante en cuanto a toda la información, de lo que te dan para leer, para estudiar, los trabajos, es muchísimo. La gente con la que te cruzás te dice, Maestra Jardinera es fácil, y así, de una patada te recibís. Y cuando entré, me di cuenta que acá no te enseñan a recortar papelitos como vos estás acostumbrada a pensar (G2). 


\section{4.b. Sobre los acontecimientos que anuncian la transformación: algunos planteos sobre el desencanto en el camino de formación}

Hay acontecimientos que son significados por las entrevistadas como de irrupción, porque incidieron en revisar concepciones y saberes, sus expectativas sobre la carrera, volver a pensar la formación en relación con unos tiempos, unos contenidos, con las formas de habitar un espacio educativo como lo es la Universidad, y sobre todo la construcción de vínculos. Las narraciones de formación con las que trabajamos, nos muestran evidencias de la importancia de los vínculos. Una de las entrevistadas nos comenta:

sentí como que otra vez volvía a vivir esa experiencia de injusticia. Me pasó, por ejemplo, que había una materia que yo había estudiado muchísimo, sabía muchísimo y no me aprobó la profesora, no sé por qué, no entendí. Viste que hay cosas que vos no entendés y como vos sos el alumno, el docente tiene la razón. (G2)

El espacio de formación se confunde con el espacio social, con la vida misma, el darse cuenta que, en este contexto, además de formarse para el ejercicio de una profesión, también hay algo del 'mundo', en términos de Masschelain y Simons (2014) que se reactualiza, aunque se presenta con otra forma. En definitiva, en este contexto de formación, se aprenden otras formas de ver y de vivir "el mundo".

Los vínculos entre docentes y estudiantes son de respeto en la mayor parte del recorrido de formación. Pero hay acontecimientos que dan cuenta de relaciones autoritarias que, en algunos casos, abonan a la construcción de cierto temor y resignación al momento de relacionarse con la o el docente. Por ejemplo, ante una situación en la que un grupo de estudiantes estaba intentando realizar un reclamo, manifiestan, "y nosotras teníamos miedo en el fondo, teníamos miedo de que se nos marcara, a ver quién es la voz que la escribió" (G1), "para qué te vas a ir a discutirle si después va a ser peor". (G2).

Esta percepción se ve matizada en una entrevistada, que atribuye a rasgos de la personalidad de algunos y algunas docentes la causa de dichos tratos, "(...) porque con su carácter, su personalidad, como que te impone, te genera un poco de ese miedo y lo que por ahí te dice, se enojó" (G1).

En este punto, entendemos que uno de los desafíos en la formación docente es "el de cómo pensar y cómo hacer de la educación ese acto político que emancipa y que asegura, con justicia, la inscripción de todos en lo público y el derecho de todos de decir y decir-se en el espacio público" (Diker y Frigerio, 2010, p. 8).

Otro tema identificado en las narraciones de formación, lo vinculamos con el acceso a la información, la presencia, el uso o la carencia de la misma va configurando modos diferentes de transitar la formación. Las entrevistadas reconocen que, tanto en el ingreso como en los primeros años, hay un desconocimiento que impacta de manera negativa en el cursado. Se desconoce el Plan de Estudios, los regímenes de cursada, los programas de las materias. En este sentido la desinformación, en algunos casos, es consecuencia de no conocer y entender ciertas lógicas propias del contexto universitario, y de este modo se acrecienta la construcción de una sensación de angustia. 
Respecto a la dimensión institucional, se reconoce como un aspecto desalentador la organización de los horarios de las distintas materias, la distribución de las materias, por ejemplo, en un primer año cursan nueve asignaturas, la misma situación se repite en el segundo año. En una de las narraciones se plantea, "como eran muchos trabajos en grupo, nosotras para alivianarnos nos dividíamos y después yo mandaba la parte que me tocó, y hoy en día hay cosas que ni llegué a leer" (G1).

También identifican como un punto central la organización interna de muchas asignaturas, por ejemplo, en la carga excesiva de contenidos, en la organización de las instancias de evaluación. Siguiendo estos sentidos, rendir materias se experimentaba como un "sacárselas" de encima:

acá en la Facultad es como que te dan mucha información y es todo apurado (...) es como que todavía no logro entender todo y es tanta información para ser todo, todavía estoy asimilando cosas que, a medida que voy leyendo se van acomodando en su lugar, y eso me pasó con la carrera. (G2)

El llegar y transitar el último año de formación es un acontecimiento relevante y vivenciado como angustiante también, según lo relatado por ambas entrevistadas. En algunos casos se produce cierta "crisis" que impacta en la decisión de continuar o no con la carrera. Esto conmueve y desestabiliza, se cuestionan las decisiones tomadas y sostenidas en todo el tiempo de formación, haciéndose presente lo que denominamos anteriormente como el segundo momento en la formación. Es el punto en el que se produce un desequilibrio que instala dudas sobre la continuidad en la carrera. Es un punto de inflexión, donde es más palpable que se está a un paso de dejar de ser estudiante para ser docente.

En el orden de lo personal también se mencionan muchos acontecimientos que obligaron a parar, a repensar. En este sentido, mientras cursan el profesorado, la vida transcurre y los acontecimientos significativos suceden, por ejemplo, el ser madre. Esto trae como consecuencia un retraso en el cursado, pero también la valoración de los tiempos dedicados a la formación.

Por último, las entrevistadas coinciden en plantear como una carencia, la preparación para el ámbito laboral. Ambas graduadas, han manifestado diferentes experiencias en relación con la inserción laboral y las dificultades que se presentan argumentando que "no encajaba" lo aprendido con la realidad. Al momento de realizar las entrevistas, ninguna contaba con cargo docente en instituciones provinciales, sí trabajaban en otros contextos, como así también en otros rubros.

\section{4.c. Sobre los acontecimientos que conmovieron el recorrido formativo y afianzan la construcción de una identidad docente}

En este punto, identificamos en las narraciones de formación el lugar relevante que tiene la experiencia de cursado en materias que proponen otros modos de conocer y otros saberes. Es en la conjunción entre la propuesta curricular y las decisiones que algunos equipos docentes toman para el desarrollo de determinadas materias, que surgen algunos planteos diferentes y que son significados de manera positiva. Por ejemplo, el Taller de la Educación Inicial es la primera materia que cursan, con una duración de tres semanas, es el primer contacto que las y los estudiantes tienen con la carrera. Este taller es reconocido como un primer 
acontecimiento importante ya que "permite tener una idea de dónde se está parado" (G1).

Asimismo, un rasgo de identidad en el plan de estudios de esta carrera es la presencia de los Talleres de Investigación, Integración y Práctica, un taller por año de duración de la carrera. Cada uno de ellos se organiza a partir de "ejes problematizadores": Desarrollo y Educación del sujeto- El rol docente y sus ámbitos de ejercicio- Contenidos curriculares y transposición didáctica - Prácticas y Residencia. (Plan de estudio Profesorado en Educación Inicial, 2000). Cuentan con instancias de cursado en el ámbito de la Facultad y de trabajo de campo intentando, de esta manera, borrar los límites que existen entre "teoría" y "práctica" en la formación.

El trabajo de campo implica el ingreso de las y los estudiantes a instituciones para realizar distintos tipos de actividades vinculadas con la investigación y la práctica docente. Ésta es una característica que nos permite decir que, en este trayecto de formación, desde el primer año de carrera, los y las estudiantes ya tienen contacto con el futuro campo laboral. En este sentido, la organización del plan de estudios irrumpe en una lógica tradicional, más academicista (donde la disciplina asume una centralidad), en el ámbito de las carreras de formación docente en la Facultad de Humanidades de nuestra Universidad.

El denominado trabajo de campo es significado de manera muy positiva por las graduadas, porque en estos espacios hacen otras lecturas sobre lo educativo, van "poniendo" teoría, autores y otras explicaciones a lo que observan en los jardines de infantes. En palabras de una de ellas, "y bueno, en la sala ya empezamos a ver las características y cómo eran los niños, empezamos a ver también de otra manera al niño, porque lo que antes era: "está jugando nomás", ahora ya tenía otro sentido" (G1).

Observamos que, lo que trasciende, en el fondo se vincula con un saber, con una forma específica de relacionarse con un conocimiento. Las asignaturas que aparecen como significativas son aquellas que imponen una forma de conocer donde el "hacer" es central y donde se aprende "poniendo el cuerpo",

y eso que decía que estaba muy vinculado a lo afectivo, eso de poner el cuerpo, el tratar de entender qué le está pasando al niño, esa sensibilidad, eso es como que me acuerdo (...) Siempre me gustó mucho esas materias donde uno podía trabajar sobre todo el vínculo. (G1)

Poner el cuerpo significa mirarse, sostener la mirada, cuando hay situaciones que las podemos vivenciar. El encuentro con el niño, en otro contexto donde la teoría adquiere otro sentido. Implica una construcción colectiva de un saber, de allí que los talleres ayudan a ir construyendo una forma de ser docente. "Poner el cuerpo" implica la construcción de un 'ir sabiendo' que es el resultado mismo de la experiencia de formación, a partir de ciertas propuestas que las pone en situación de correrse de la seguridad de la lectura individual de un libro, de un apunte, de un dossier, para dejar entrar lo corporal, las sensaciones, lo colectivo porque es con la otra, con el otro, que se comienza a mover estructuras que, con el tiempo, se convierten en estructuras de comprensión y acción. 


\section{4.d. Estudiar en la Universidad}

En las experiencias de formación narradas se puso en evidencia que, el hecho de estar en la Universidad es una decisión de vida. En ambos casos, iniciar la carrera de profesorado en Educación Inicial fue pensado en este único espacio, "pero cuando decidí estudiar, yo dije me voy a estudiar en la UNNE, no importa lo que cueste" (G2).

El habitar la Universidad conjuga tensiones, por un lado, lo que sucede allí, "todo es apurado" y "hay mucha información". Pero esto convive con la fuerte carga que se otorga a esta formación, al título universitario, como dice una de ellas, "da prestigio". Para uno de los casos implicó ser la primera universitaria en la familia.

Reconocen que estudiar en la Facultad favorece a la construcción de una "mirada crítica", entendiéndola como, "una herramienta que me dio la Facu, poner sobre la mesa las cosas que me están pasando, los sentimientos, el cómo estoy, cómo estoy ante la sala, delante de los nenes y también qué le estoy pidiendo a ellos" (G1). Aunque luego reconozcan que es muy difícil sostener esta mirada en los distintos puestos laborales. Pero comprendemos que,

Algunas de estas tesis expresan los alcances del proceso de apropiación subjetiva de la institución en la trama de identificaciones inestables pero dotadas de sensibilidad y de aprendizajes; otras aluden a rasgos particulares de las culturas institucionales a la vez que indican una perspectiva crítica sobre las dinámicas académicas. (Carli, 2012, p. 251)

Formación y universidad conjugan, lo que Masschelein y Simon (2014) nos invitan a pensar, la formación como una apertura al mundo, algo nuevo forma parte de nuestro mundo, por el cual nos implicamos y comprometemos y de este modo, algo se transforma.

\section{A modo de cierre}

Cada camino de formación esconde una aventura particular que lo hace único. Por lo tanto, sumergirnos en este espacio de experiencias narradas nos abre algunas puertas para comprender, desde otras miradas, a la formación docente en el ámbito de una Facultad, de una Universidad pública. Cullen (2008) plantea que el desafío se encuentra en poder mirar la palabra como don, un regalo, "y entonces darla y tomarla" (p. 7). Y este es nuestro desafío, tomar la palabra que nos fue dada y hacerla nuevo rumbo de viaje. Por eso, este punteo de ideas queda a modo de mapa que nos conducirá por nuevos senderos.

A lo largo del trabajo, nos dejamos conducir por aquellos acontecimientos significativos, narrados por las graduadas, que nos llevaron a delinear algunas ideas centrales. La primera está vinculada con los motivos y expectativas que plantearon, reconociéndose como principales la preferencia por el trabajo con niños y el reconocimiento de la Universidad Pública como la mejor opción para el inicio de su formación docente. Motivos que se construyeron desde el deseo inicial que marcó el andar, y que se manifestaban en frases como "ser profesionales formadas en la universidad". Este deseo y la decisión sobre su formación es, desde nuestro punto de vista y tomando las palabras de Larrosa, el punto mágico donde nacen los demás círculos, es decir, el fuego que recrea constantemente a la docente, a la profesional. 
Se narraron acontecimientos vividos con cierta angustia, que por momentos son significados como obstáculos, pero que sin dudas abonaron al proceso de construcción y deconstrucción realizado en el tiempo de formación. Estas vivencias impactaron en una forma de entender a la formación como "una carrera", en la cual se hace lo que sea por llegar a la meta, incluso ceder ante situaciones consideradas injustas, por miedo a que expresarse les deje fuera del premio. Sin embargo, este aparente desencanto, anuncia la esperada transformación, aquella que permitió a nuestras protagonistas dejar el lugar de estudiantes y darse la autoridad, primero como estudiante, para luego ir construyendo la de profesional.

Aquellos acontecimientos vividos positivamente, aquellos que afianzaron la construcción de una identidad docente, se vinculan con la construcción de un saber, donde se valora principalmente aquellos conocimientos que irrumpen una tradición academicista y están más vinculados con una práctica, con un hacer. Un saber al que "hay que ponerle el cuerpo" y que se construye de manera colectiva. Éste es un proceso que deja una huella imborrable.

En este sentido, seguimos pensando la formación como una experiencia de apertura a otros mundos posibles, la cual implica una transformación, un camino que se reinventa día a día configurando un tiempo de formación que como expresamos, no es lineal, pero sí tiene un origen común, que es la decisión de iniciar la formación docente guiado por el deseo de ser una profesional crítica.

La Universidad pública, como contexto de formación, favoreció a la construcción de ciertas características en ese camino elegido. En este sentido, queremos rescatar el lugar de la construcción colectiva del conocimiento y de los vínculos con otros y otras protagonistas del trayecto de formación, y que son una clara evidencia de la transformación experimentada por las graduadas.

Finalmente, este camino que comenzamos a andar, teniendo como brújula las voces de las graduadas, significó acercarnos a algunos sentidos de formación construidos por quienes han transitado este recorrido por la Universidad, también descubrir que aquellos acontecimientos, incluso los que nos gustaría que hayan sido diferentes, se convierten en la "partícula de impureza" por medio de la cual, la experiencia concentra, como una perla, su propia autoridad. "Porque la experiencia no tiene su correlato necesario en el conocimiento, sino en la autoridad, es decir en la palabra y el relato" (Agamben, 2004, citado en Alliaud, 2010, p. 151).

\section{Referencias bibliográficas}

Alliaud, A. (2010). Experiencia, saber y formación. Revista de Educación,1(1),141-157. Recuperado de https://fh.mdp.edu.ar/revistas/index.php/r_educ/article/view/11/55

Bárcena, F. y Mélich J. (2014). La educación como acontecimiento ético. Natalidad, narración y hospitalidad. Buenos Aires, Argentina: Miño y Dávila.

Bolívar, J. y Fernández, M. (2001). La investigación biográfico- narrativa en educación. Enfoque y metodología. Madrid, España: La Muralla.

Borda, V. y Ramirez, I. (2019). Investigar la formación docente para la Educación Inicial. Abordaje desde la experiencia de estudiantes. Ponencia presentada en las IV Jornadas de Intercambio de la Producción Científica en Humanidades y 
Ciencias Sociales, Facultad de Humanidades, Instituto de Investigaciones Geohistóricas, CONICET-UNNE y el Centro de Estudios Sociales- UNNE, Resistencia, Chaco, Argentina.

Carli, S. (2002). Niñez, pedagogía y política. Transformaciones acerca de la infancia en la historia de la educación argentina entre 1880-1955. Buenos Aires, Argentina: Miño y Dávila.

Carli, S. (2012). El estudiante universitario. Hacia una historia del presente de la educación pública. Buenos Aires, Argentina: Siglo XXI.

Comisión Mixta Educación Inicial (2019). Proceso Participativo de Revisión de los Planes de Estudio. Facultad de Humanidades, UNNE. Recuperado de https://hum.unne.edu.ar/academica/jornada/informes com/inicial.pdf

Cullen, C. (2008). ¿Infancias o facundias interpelantes? Volver a pensar la niñez en el siglo XXI, porque lo mismo no es lo igual. En G. Palacín, N. Burgos y N. Montaiuti (Comps.). Repensar la niñez en el siglo XXI. Conferencia llevada a cabo en $X$ Encuentro Nacional Universitario de carreras de Educación Infantil y II Congreso Internacional, R.E.D.U.E.I. Mendoza, Argentina.

De Souza, E. (2008). Las historias de vida y las prácticas de formación: apuntamientos sobre pesquisa, enseño y formación. Ponencia presentada en VII Seminario Red Estrado: Nuevas regulaciones en América Latina. Facultad de Filosofía y Letras, Universidad de Buenos Aires. Buenos Aires, Argentina.

Dicker, G. y Terigi, F. (2003). La formación de maestros y profesores: hoja de ruta. Buenos Aires, Argentina: Paidós.

Frigerio, G. y Diker, G. (Comps.) (2010). Educar: ese acto político. Entre Ríos, Argentina: Fundación La Hendija.

Larrosa, J. (2011). Experiencia y Alteridad en Educación. En C. Skliar y J. Larrosa (Comps.) Experiencia y alteridad en educación (pp. 13-44). Rosario, Argentina: Homo Sapiens/FLACSO.

Larrosa, J. (2000). Pedagogía Profana. Estudios sobre Lenguaje, Subjetividad y Formación. Buenos Aires, Argentina: Novedades Educativas.

Masschelein, J. \& Simons, M. (2014). Defensa de la escuela. Una cuestión pública. Buenos Aires, Argentina: Miño y Dávila.

Musin, A., Ramirez, I. y Almirón, V. (2016). Historia de la formación de docentes para el nivel inicial en el Chaco: la conformación de la carrera de profesorado de Educación Pre- Elemental en la Universidad Nacional del Nordeste (19731982). Revista de la Escuela de Ciencias de la Educación, 12(11), 65-78. https://doi.org/10.35305/rece.v1i11.250

Ponce, R. (2012). Los debates de la educación inicial en la Argentina. Persistencias, transformaciones y resignificaciones a lo largo de la historia. En A. Malajovich (Comp.). Experiencias y reflexiones sobre la educación inicial. Una mirada latinoamericana (pp. 19-101). Ciudadela, Argentina: Siglo XXI. 
Rivas Flores, J. y Leite Méndez, A. (2014). Vida y experiencia escolar. Biografías escolares. En J. Rivas Flores, A. Leite Méndez y Prados Mejías, E. (Coords.) Profesorado, escuela y diversidad. La realidad educativa desde una mirada narrativa (pp. 59- 74). Málaga, España: Aljibe.

Sirvent, M. T. (2005). El Proceso de Investigación. Cuadernos de la Oficina de Publicaciones de la Facultad de Filosofía y Letras (Opfyl). Recuperado de http://postitulo.socioeducativa.infd.edu.ar/archivos/repositorio/500/718/Sirvent El proceso de investigacion.pdf

Sverdlick, I. (2007). La investigación educativa como instrumento de acción, de formación y de cambio. En I. Sverdlick (Comp.) La investigación educativa. Una herramienta de conocimiento y acción (pp. 15- 46). Buenos Aires, Argentina: Noveduc.

Toro, J. (2005). Educar con co-razón. Bilbao, España: Desclèe De Brouwer.

Vasilachis De Gialdino, I. (2006). Estrategias de Investigación Cualitativa. Barcelona, España: GEDISA. 